\title{
żlndios amigos o pobladores criollos? Las solicitudes de tierras de los Maicá en Buenos Aires a principios de 1860s
}

\author{
¿Friendly indians or creole settlers? Maicas' land requests in Buenos Aires at the beginning of 1860s
}

\author{
Victoria Pedrotta \\ CONICET, Universidad Maimónides y Fundación Azara. \\ Facultad de Ciencias Naturales y Museo, \\ Universidad Nacional de La Plata, Argentina \\ vpedrotta@conicet.gov.ar \\ Sol Lanteri \\ CONICET, Instituto de Historia Argentina y Americana \\ "Dr. Emilio Ravignani", Facultad de Filosofía y Letras, \\ Universidad de Buenos Aires, Argentina \\ sol_lanteri@conicet.gov.ar
}

\begin{abstract}
Resumen:
Examinamos las solicitudes de tierras a título individual efectuadas por el cacique Juan Maicá, su hijo Mariano Maicá y su lenguaraz Antonio Medina durante 1861, en plena revisión liberal post-rosista hacia finales del período de enfrentamiento entre el Estado de Buenos Aires y la Confederación hasta 1862. Partimos de la importancia de considerar la relación entre armas, tierras y política en la frontera, especialmente en esta coyuntura bélica e institucional. Como intentaremos demostrar mediante el contraste de fuentes inéditas y editadas complementarias, el caso que analizamos de forma original permite destacar la capacidad de agencia indígena y el accionar de sus líderes étnicos en torno a la gestión de la tierra frente al Estado en construcción y la sociedad criolla, el conocimiento y la utilización de los marcos normativos en su provecho y el rol de intermediario del sargento Juan Cornell. Consideramos además algunos puntos en común con solicitudes indígenas de tierras en otros sectores de la frontera bonaerense.

Palabras ClaVe: Frontera sur de Buenos Aires, Década de 1852-1862, "indios amigos", Ssolicitudes de tierras, Armas y política.
\end{abstract}

\section{Abstract:}

We examine individual land requests by ethnic chief Juan Maicá, his son Mariano Maicá and the "lenguaraz" (translator) Antonio Medina in 1861, in the midst of the liberal revision after the Rosas government towards the end of the confrontation between the State of Buenos Aires and the Confederation until 1862. We support the importance of considering jointly the relationship among arms, lands and politics in the frontier, especially in this military and institutional conjuncture. As we will try to demonstrate contrasting complementary published and unpublished sources, the case we originally analize allow us to point out the indigenous agency and the ethnic leaders' actions in relation to the State under building and the Creole society, the knowledge and use of the normative frameworks to their advantage and the intermediary role of Sargent Juan Cornell. Moreover, we consider some similarities with indigenous' land requests in other parts of the Buenos Aires frontier.

KEYWORDS: Buenos Aires southern frontier, Decade of 1852-1862, "Friendly indians", Land requests, Armed service and politics.

\section{INTRODUCCIÓN}

En los últimos años, las formas de acceso y uso del suelo y otros recursos por parte de diversas poblaciones indígenas, y demás sectores sociales, de los ámbitos rurales o fronterizos de América Latina han sido revisitadas, y esto ha echado luz sobre los cambios, transformaciones o continuidades de los grupos, sus prácticas y estrategias al calor de los procesos de "modernización liberal” y la relación con los diferentes 
contextos sociohistóricos durante la formación de los Estados-nación modernos (entre otros, Escobar Ohmstede, Medina Bustos y Trejo Contreras, 2015; de Jong y Escobar Ohmstede, 2016; Motta y Piccolo 2017a, 2017b).

Respecto de la Argentina, contamos con varios estudios sobre las regiones del Noroeste, el Litoral y Pampeana (por ejemplo AA.VV., 2015), así como vinculados a las diferentes zonas de la frontera bonaerense (entre otros, Lanteri et al., 2011; de Jong, 2015; Literas y Barbuto, 2015; Literas, 2015; Cordero, 2017; Martinelli y Acosta, 2016; Martinelli, 2017; Yuln, 2017). Estas investigaciones, realizadas desde diferentes disciplinas humanas y sociales como la Historia, la Antropología Social, la Arqueología Histórica o la Arquitectura Histórica, han develado una gran diversidad de situaciones respecto a las formas de gestión, acceso y traspaso del recurso, usos comunales o privados de las tierras, el surgimiento de nuevas territorialidades, etc., en función de factores como la estructura y relaciones de poder intra e intertribales, la complejidad de las relaciones interétnicas con los sucesivos gobiernos y las características de los ecosistemas, contemplando los cambios y/o continuidades entre la década de 1850 y las posteriores una vez unificado el Estado a partir de 1862 .

En este marco, en investigaciones individuales y conjuntas interdisciplinarias, hemos indagado distintos aspectos concernientes al acceso, poblamiento, territorialidad y formas de entrega del recurso a distintos grupos de "indios amigos" de la frontera centro-sud bonaerense, analizando diferentes formas de adjudicación de tierras y solares urbanos especialmente durante los gobiernos de Rosas (1829-1852) y la conflictiva década de 1850 (Lanteri, 2013, 2017; Lanteri y Pedrotta, 2012, 2018, entre otros).

En el presente trabajo nos proponemos examinar las solicitudes de tierras a título individual por parte del cacique Juan Maicá, su hijo Mariano Maicá y su lenguaraz, Antonio Medina, en la frontera sur de Buenos Aires durante el año 1861. Se trata de solicitudes de "suertes de estancia", que fueron efectuadas en pleno proceso de revisión liberal post-rosista en materia de tierras públicas, hacia finales del conflictivo período de enfrentamiento entre el Estado de Buenos Aires y la Confederación Argentina abierto en 1852 hasta la primera presidencia de la república unificada en 1862. Las fuentes que utilizamos son, primeramente, los tres expedientes de tierras que contienen dichas solicitudes -no estudiados hasta el momento- junto con fuentes complementarias, que incluyen correspondencia oficial edita e inédita, expedientes de tierras, duplicados de mensuras, informes y registros gráficos elaborados por autoridades locales, registros parroquiales, testimonios de actores contemporáneos, entre otras.

Partimos de la premisa de la importancia de considerar de forma conjunta la relación entre armas, tierras y política en la frontera, especialmente en esta particular coyuntura bélica e institucional (Lanteri y Pedrotta, 2018). Como intentaremos demostrar, los casos que examinamos en detalle de forma original nos permiten, por un lado, destacar la capacidad de agencia de los propios grupos indígenas y sus principales líderes étnicos en cuanto a la gestión de las tierras frente al Estado en construcción y la sociedad criolla, así como el conocimiento y la utilización de los marcos normativos e institucionales en su provecho. Por otro, conocer el accionar oficial con respecto a estos pedidos, que constituyen el primer antecedente de solicitudes indígenas de tierras según la modalidad de "donaciones condicionadas", que había sido implementada durante el gobierno de J. M. de Rosas en la región (Lanteri, 2011).

De tal forma, en primer lugar pasaremos revista a los principales sucesos y procesos de la frontera bonaerense desde la caída de Rosas hasta la unificación nacional, para luego detenernos en los antecedentes de los Maicá y su inclusión como "indios amigos" de la frontera, y, finalmente, el proceso concreto de solicitudes de tierras a título individual, considerando sus principales características, agentes e instituciones involucradas y sus resultados. Asimismo, tomamos en cuenta algunos puntos en común que presenta el caso con distintas solicitudes indígenas de tierras en otros sectores de la frontera. 


\section{LA Frontera SUR DE Buenos Aires hasta LA UNIFICACIÓN NACIONAL}

La derrota de Juan Manuel de Rosas en 1852 en la batalla de Caseros y sucesos posteriores marcaron el inicio de un período de ruptura entre el Estado de Buenos Aires y la Confederación Argentina, que se extendió hasta 1861, cuando, tras otro hecho de armas -la batalla de Pavón-, se generaron las condiciones para la ulterior unificación del Estado argentino. Ese período de separación y enfrentamiento entre Buenos Aires y la Confederación tuvo gran repercusión en los espacios fronterizos, a la vez que impactó sobre los vínculos entre los distintos gobiernos y el mundo indígena, cuyas relaciones se caracterizaron por un abanico variado, que fue desde la lealtad militar constante de ciertos caciques hasta la alternancia estratégica de alianzas volátiles con uno y otro bando criollo en pugna. En la frontera bonaerense, además, los cambios oscilantes en la política indígena, que sucedieron en los primeros años de la coyuntura post-rosista, derivaron en un ciclo de violencia interétnica, el cual se agudizó especialmente entre 1853 y 1855 . Sin la capacidad bélica ni los medios para someter militarmente a los grupos indígenas sublevados, el Estado de Buenos Aires debió acudir a una política de pacificación que se cimentó en la firma de tratados de paz con los caciques Juan Catriel y Juan Manuel Cachul, del centro bonaerense -en octubre de 1856-, y con el cacique José María B. Llanquetruz, del extremo sur provincial -en mayo de 1857-, sumados a diversas propuestas de paz que buscaron aquietar a Juan Calfucurá en esos años (Lanteri y Pedrotta, 2018; Levaggi, 2000; Ratto, 2015).

El tratado concertado con los caciques Catriel y Cachul constituye -a diferencia de la oralidad propia de la política indígena de Rosas- el primer reconocimiento oficial mediante acuerdo escrito de la "legítima propiedad" sobre una extensión de tierras a una tribu ${ }^{1}$ indígena durante el período independiente. En el también se acordó restablecer el comercio entre las partes, reanudar el racionamiento estatal a los "indios amigos" y otorgarles nombramientos militares con sus respectivas remuneraciones, entre otros puntos (Lanteri y Pedrotta, 2012, 2018). Paralelamente, el coronel Manuel Escalada, quien tuvo a su cargo buena parte de la negociación del tratado, gestionó ante la Corporación Municipal de Azul la compra de tierras adyacentes al pueblo para ser distribuidas entre los indígenas. A partir de una traza en damero con una plaza central, esos terrenos se cuadricularon en manzanas divididas en 100 solares de 50 varas de frente por 50 de fondo, y pasaron a formar el barrio de Villa Fidelidad. Allí se asentaron muchos integrantes de la agrupación al mando del cacique Maicá, adjudicatarios principales del reparto inicial de solares. Este caso representa una modalidad bastante inédita de otorgamiento de tierras a indígenas, porque no fue colectiva sino a título individual-familiar, porque se trata de un sector dentro de la traza del pueblo, y porque la organización en damero no reconoce antecedentes en los patrones de ordenamiento espacial indígena, previos ni contemporáneos (Lanteri y Pedrotta, 2016, 2018).

\section{FIGURA 1}

Reorganización de la Frontera sur de Buenos Aires de 1859 y lugares aludidos en el texto

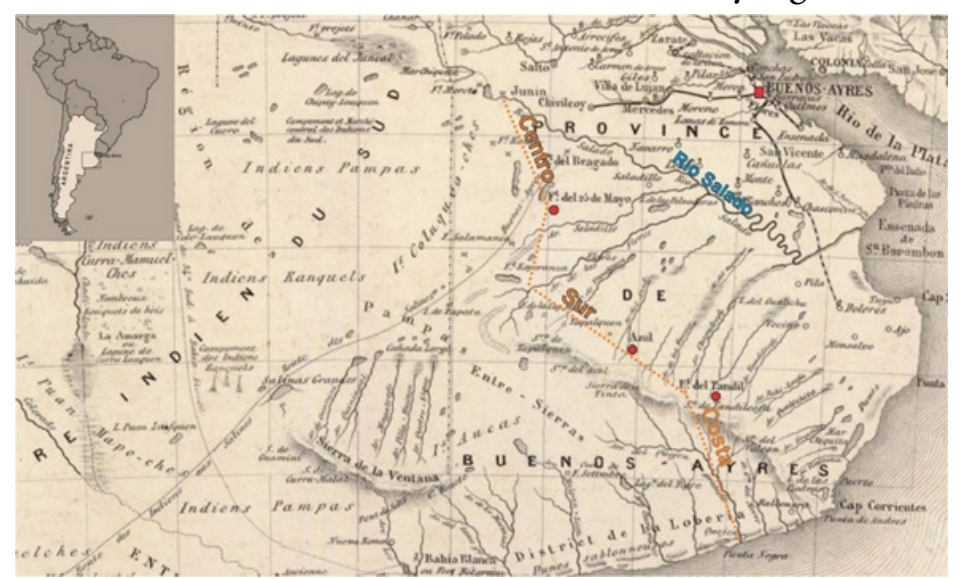

Fuente: elaboración propia sobre la Carte de la Confederation Argentine, de M. De Moussy, 1867. 
Con la gobernación de Valentín Alsina, iniciada en mayo de 1857, se reorganizó la Guardia Nacional de Campaña y el Ejército de Operaciones del Sud, y se dispuso el avance de la línea de fronteras, aunque esta última no se tradujo en la incorporación de nuevas tierras sino más bien reafirmaba el territorio entonces ocupado. Dicho ejército, al mando de Nicolás Granada, integrado por las fuerzas que guarnecían los fuertes y fortines de la línea de frontera, por guardias nacionales movilizados y por "indios amigos" del centro de la provincia, quedó constituido por dos divisiones: la primera división en Azul y la segunda en Bahía Blanca, bajo las órdenes de los coroneles Emilio Conesa y Wenceslao Paunero, respectivamente (Walther, 1973, p. 302-303).

No obstante la pacificación lograda en ciertos sectores de la frontera, en otros sectores los ataques y robos por parte de grupos indígenas hostiles continuaron durante 1857 y $1858 .{ }^{2}$ En una situación crítica de desabastecimiento, escasez de hombres y armamento entre las fuerzas del gobierno, agravada por la fuerte sequía de 1858, el teniente coronel Ignacio Rivas fue promovido a jefe de la Frontera Sud. Una de sus tareas principales fue la de lograr el apoyo de la mayor cantidad de caciques posible de cara a la inminente guerra abierta entre Buenos Aires y la Confederación. ${ }^{3}$ El sostén de las fuerzas lideradas por J. Catriel y J. M. Cachul fue fundamental entonces para la defensa de la frontera, las estancias y los poblados criollos. ${ }^{4}$

En octubre de 1859 el ejército de la Confederación Argentina venció al del Estado de Buenos Aires en la batalla de Cepeda. En esa coyuntura, ante el traslado del ejército al frente de combate, que contaba con el refuerzo de 430 lanceros de Juan Catriel, la protección del pueblo y fuerte de Azul había sido confiada a dicho cacique como "Comandante General" (Catriel, 1859). De hecho, Azul fue atacado por fuerzas al mando de Calfucurá y de Pedro Rosas y Belgrano, quien logró ocupar temporariamente el pueblo en noviembre de ese año, hasta la liberación del mismo por el coronel Nicolás Ocampo “con los derrotados de Cepeda" (Del Valle, 1926, p. 134-135).

El triunfo definitivo de Buenos Aires en la batalla de Pavón, en septiembre de 1861, dio inicio al período de unificación política del Estado bajo la presidencia de Bartolomé Mitre (1862-1868). La frontera bonaerense había sido reorganizada en 1859 con la creación de los Departamentos Sur -desde al arroyo Chapaleofú hasta el fortín Esperanza- y Costa -desde dicho arroyo hasta la costa oceánica-, al mando de los coroneles Ignacio Rivas y Benito Machado, respectivamente (Figura 1). Ello no obedeció a avances territoriales significativos, sino más bien a la búsqueda de una estructura defensiva efectiva para contener a los malones indígenas que se sucedieron desde la caída de Rosas. ${ }^{5}$

\section{El liNAJE de CACiQues MaICÁ}

Acorde la biografía efectuada por el padre Hux (1993, p. 128-129), "los Maicá o Maycá o Mahicá” aparecen en la documentación desde 1820, como referentes ante los enviados del gobierno y gestores de varias negociaciones y encuentros diplomáticos inter e intraétnicos. Junto con el cacique Antuen, al mando de unos 100 lanceros, acompañaron a Rosas durante la campaña militar de 1833-1834; según algunos estudiosos, para entonces eran "absolutamente independientes de los catrieleros" (Sarramone, 1993, p. 180, cursiva nuestra). ${ }^{6}$ En esa misma década, las tolderías de los caciques Maicá y Peti estaban situadas en la cuenca del arroyo Sauce Corto, en el sur bonaerense, y fueron atacadas por fuerzas comandadas por Manuel Delgado y Narciso del Valle. Esta derrota -que fue reseada como la "Jornada del Sauce Corto" en las memorias de Juan Cornell (1995 [1864], p. 46)-, y la consiguiente necesidad de poner a salvo a sus familias, determinda rendiciónde dichos caciques y su posterior incorporacióml "negocio pacífico".7 De este modo, para la dcada de 1840, ambos aparecen en los listados de "indios amigos" de Azul que reciban el racionamiento gubernamental (fuentes del Archivo Histrico E. Squirru de Azul citadas por Ronco, 1930, p. 134; Hux, 1993, p. 130 y Sarramone, 1993, p. 139 nota 357). 
A partir del traslado del grupo a Azul, los lanceros liderados por Maicá pasaron a formar parte de las fuerzas militares que dependían de la Comandancia de la Frontera Sur, sin que hayamos encontrado hasta el momento - a diferencia de lo ocurrido con numerosos caciques principales, como Catriel, Cachul y Calfucurá, o secundarios, como Chipitruz y Manuel Grande- registros de sublevaciones o de alianzas ocasionales con grupos enemigos del gobierno, sino todo lo contrario. En contraste con otros grupos de "indios amigos" que prestaron servicios militares en diferentes sectores de la frontera sur -sin que ello conllevara su subordinación militar efectiva (de Jong 2011, p. 33)-, el contingente de los guerreros de Maicá formó parte del ejército regular como cuerpos de línea o guardias nacionales, bajo las órdenes del jefe de turno de la mencionada comandancia militar sita en Azul. Según nuestra información, su apoyo no parece haber dependido exclusivamente de las alianzas previas dentro del campo indígena ni de las ventajas que podían ofrecer las cambiantes circunstancias en la frontera. Basta con mencionar, a modo de ejemplo, la participación de los Maicá junto a las fuerzas estatales en los combates de Sierra Chica (1855), San Jacinto (1855), Sol de Mayo (1857), Cristiano Muerto (1857), y en la defensa de la plaza de Azul ante el ataque emprendido por Calfucurá junto a Rosas y Belgrano en noviembre de 1859, entre otros episodios bélicos menores (Arena et al., 1967; González, 1967; Hux, 1993).

En trabajos anteriores hemos estudiado la génesis histórica de Villa Fidelidad, destacando el vínculo entre la prestación de servicios militares y la entrega de tierras, así como la férrea lealtad que demostraron los lanceros de Maicá -que fueron incorporados al Regimiento de Coraceros N. 2 al mando del coronel Manuel Ocampo (Arena et al., 1967, p. 209-212; Barbará, 1856, p. 46; Hux, 1993, p. 130)-durante los enfrentamientos con los grupos que se habían sumado a la confederación liderada por Calfucurá en la crítica coyuntura post-rosista y el otorgamiento de los solares en propiedad individual que dieron origen a la mencionada villa azuleña a partir de 1856 (Lanteri, 2017; Lanteri y Pedrotta, 2018). En particular, se destaca Mariano Maicá, quien fue el que tuvo mayor recorrido dentro de la carrera militar en el ámbito local (alcanzó el grado de sargento mayor). Esta trayectoria presenta puntos en común con Martín Rondeau, jefe borogano asentado en el oeste provincial, quien pasó de ser soldado a oficial, para luego reportar como teniente primero de la Caballería del Ejército de Línea, a cargo de la compañía de “indios amigos” (Literas, 2015, p. 63).

Además de la gestión y obtención de dichas extensiones de tierra de forma autónoma y de su trato directo y presumiblemente personal con el coronel Escalada, en el contexto de los tratados de paz firmados entre 1856 y 1857, durante los años siguientes hallamos numerosas fuentes que indican que Maicá gestionaba y recibía su paga militar y sus raciones de forma totalmente independiente de los caciques Catriel y Cachul. Por ejemplo, en los meses previos a la batalla de Cepeda las autoridades militares de Azul pusieron especial cuidado en que los lanceros de Maicá recibieran su remuneración y armamento, así como 500 yeguas que habían sido reunidas ex profeso por los jueces de paz de varios partidos de la campaña y fueron entregadas al capitanejo Roque Maicá, hijo del cacique, en persona (Expediente, 1858; Comandancia, 1859). Esta dinámica también ha quedado documentada para la década de 1860, incluso en ocasiones con la intervención del propio ministro de Guerra y Marina, el general Juan Gelly y Obes $(1860,1861)$.

\section{LAS SOLICITUDES DE TIERRAS}

Como hemos visto, los Maicá tenían un largo recorrido como “indios amigos” en la frontera y con un arraigo territorial importante, particularmente en la zona de Azul desde 1830, cristalizado además con el establecimiento de Villa Fidelidad en 1856 y su participación militar regular guarneciendo el punto. Como ya se señaló, la donación de solares en la traza urbana del pueblo realizada por el general Escalada, con el aval del Estado de Buenos Aires, principalmente a este grupo indígena constituyó una novedad en el contexto bonaerense (Lanteri, 2017; Lanteri y Pedrotta, 2018).

En este mismo sentido pueden considerarse las solicitudes de tierras a título individual en el partido de Azul realizadas unos años después por el cacique Juan Maicá, por su hijo Mariano y por el lenguaraz Antonio 
Medina al gobierno con la intermediación del sargento mayor Juan Cornell. Este último era una autoridad reconocida en la frontera, y había sido designado en 1859 por Dalmacio Vélez Sarsfield, entonces ministro de Gobierno, para realizar un informe sobre las suertes del Azul, al que anexó un plano elaborado a mano (Cornell, 1859a, 1859b). ${ }^{8}$ Estos documentos constituyen las fuentes más valiosas sobre el tema. Ambos, el informe y el plano, fueron elevados al Departamento Topográfico (en adelante DT) ese mismo año y contienen información detallada sobre los pobladores, los antecedentes y las características de cada terreno, entre otros elementos.

Según la información con que contamos, es la primera vez que un pedido de tierras de parte de indígenas al Estado en la frontera centro-sur se llevó a cabo mediante un intermediario criollo, y no de forma directa, como sucedió en otras secciones de la frontera provincial. Es dable destacar también la rapidez que implicó todo el proceso de tramitación. Desde que se otorgaron los poderes de los interesados a Cornell, se hizo la presentación formal al Superior Gobierno, y se expidió la respuesta oficial pasaron solo dos meses -de febrero a abril de 1861-, aun con varias instituciones, autoridades e instancias intermedias implicadas desde la frontera hasta la ciudad de Buenos Aires (Cornell, 1861). Veamos con más detalle todo a continuación.

En el pueblo de Azul, el 4 de febrero de 1861, "el Sargento mayor en actual Servicio en el ejército que guarnece este punto Don Mariano Maicá" compareció ante el juez de Paz Juan M. Rivero -por falta de escribano- $y$ testigos de actuación para otorgar un poder al

Sargento mayor de Línea Don Juan Cornell vecino de la ciudad de Buenos Ayres residente actualmente en este para que á su nombre y representando su persona acciones y derechos como si el mismo fuere se presente formalmente al Superior Gobierno- solicitando se le conceda para poblar con arreglo á las leyes de la materia; una suerte de estancia donde la hubiera valdia en este Partido del Azul, haciendo para el logro de esta concesion- cuantas diligencias concidere necesarias. ${ }^{9}$

La fuente agrega que "no firmó porque dijo no saber y lo hizo á su ruego uno de los testigos presentes", José Flores, siendo el otro testigo de actuación del poder (que firmó el acta pero no a nombre de Maicá) Basilio Vásquez. A continuación figura la carta escrita y firmada por el propio Juan Cornell, en Buenos Aires el 5 de marzo del mismo año, es decir, un mes luego de recibir el poder de Maicá. En ella -a nombre y en representación del anterior y destacando que el mismo se encontraba en "servicio en el Azul"- sostuvo (Cornell, 1861, cursiva nuestra):

que mi representado se interesa en poblar un establecimiento de estancia en el partido de Azul y pa ello me autoriza á fin de que denuncie y pida á su nombre en propiedad una suerte de estancia compuesta de media legua de frente por una y media de fondo en él terreno valdio que fue destinado antes de ahora $p^{a}$. la repartición de suertes en aquel partido según el decreto de 1829.

A ello agregó: "cuyo terreno, esto es la suerte que solicito, se registra de la parte occidental del Arroyo Azul en mi plano Croquis que eleve al conocimiento de V.E. y que hoy se halla en el Departamento Topográfico”. Con respecto a la ubicación, sostenía que su solicitud, en caso de tener lugar

debera medirse haciendo frente al Sud-Este á la suerte $\mathrm{N}^{\circ} 30$ de mi mencionado plano que esta en segunda escala de la que corresponde a la testamentaria de Alvarado $\mathrm{N}^{\circ} 106$ sobre la costa del Arroyo, asi como la que solicito quedara en tercera escala lindando por el Sud-Oeste con la q. también solicito pa. el casique Mayca padre de mi representado - por el Nor-Oeste con los terrenos o cerca de ellos que corresponden a Villamayor, y á Dn Luys Goya taperas de Jurado-por el Nor-Este con la denuncia del lenguaraz del casique Mayca Antonio Medina á quien asimismo yo represento.

Finalmente, terminaba la misiva pidiendo se agregara su solicitud a la del cacique Maicá hasta que fuera desglosado el trámite (Cornell, 1861, cursiva nuestra). Esta carta fue certificada ante el escribano de Gobierno Juan Madera, con fecha 8 de marzo de ese año y transferida la solicitud al Departamento Topográfico al día siguiente. Apenas 10 días después, el 19 de marzo, el DT se expidió "según los registros qe obran en esta Oficina" brindando información sobre linderos, propietarios y otros del terreno para que "el Sr Gefe determinará lo que corresponda”, con las firmas de Salas, Moreno, Fernández y Malaver. El 23 de marzo, 
Antonino Marcó del Pont, jefe de la Oficina de Tierras Públicas y Bienes del Estado, firmó un decreto al respecto, al que dio fe el escribano Madera.

A continuación, en el expediente figura, con número consiguiente 659, el poder -de idéntica forma y naturaleza, lugar y fecha, testigos y autoridad que el anterior- que el cacique Juan Maicá, "actualmente a lás inmediatas órdenes del Señor Comandante en Jefe de la Frontera del Sud", otorgó a Cornell, "vecino de este Pueblo y empleado por el Superior Gobierno", para que a su nombre solicitara al Superior Gobierno la concesión para ocupar una suerte de estancia en el Azul, firmando también en su nombre por no saber y como testigo José Flores.

En mismo lugar y fecha que la solicitud anterior (Buenos Aires, 5/03/1861), Juan Cornell, en nombre y representación del cacique Maicá, "que esta en actual servicio con su tribu en el Azul”, pidió en propiedad una suerte de estancia para formar un establecimiento "denunciando el campo valdio que hay á la parte occidental del Arroyo Azul que se comprende [d]entro del Area en la que se ha hecho la reparticion de las suertes de estancia en dho Partido". El terreno, de iguales dimensiones (una suerte de media legua de frente por una y media de fondo), fue solicitado de forma contigua al pedido de Mariano Maicá y registrado en su croquis, debiendo medirse su frente de media legua rumbo sudeste a la suerte $n^{\circ} 31$ de dicho plano, con información detallada sobre los linderos en todos los ejes cardinales. La solicitud corrió igual tramitación que la anterior en todos sus pasos y autoridades, brindando el DT la misma información que el terreno de Mariano por ser colindantes.

Finalmente, la última solicitud del expediente, la n 660, es la que realizó Cornell a nombre del lenguaraz del cacique Maicá, "Don” Antonio Medina y "vecino de este pueblo" (Azul), quien le había otorgado previamente un poder en idénticas condiciones que los dos anteriores para que hiciera la presentación formal al Superior Gobierno "solicitando le conceda la posesion de una suerte de estancia donde la hubiera valdia en el partido del Azul”. Cornell llevó esto a cabo como lo había hecho con los pedidos anteriores aduciendo que su representado

quiere poblarse formando un establecimiento de estancia á la par de las suertes que con esta fha [5/03/1861] ha solicitado [...] y al efecto me ha otorgado poder especial $\mathrm{p}^{\mathrm{a}}$. pedir en propiedad y á su nombre una suerte de estancia en el Partido Azul [d]entro de los terrenos ó Area que de antes de ahora se han repartido las suertes con arreglo al decreto de 19 de Nbre de 1829.

Cornell también dio la ubicación precisa del terreno solicitado en el croquis que había realizado y elevado al DT dos años antes, sosteniendo, como inigualable conocedor del poblamiento y la topografía del lugar, que: "El campo valdío que existe y denuncio [...] biene á quedar á los fondos de la suerte $n^{\circ} 29$ de dho plano a la parte occidental del Arroyo Azul, si es que no bubiese en ese parage algún antecedente de mejor derecho-y en caso de no baberlo y $V$. E. accede á esta solicitud..." iba a ser colindante a las parcelas pedidas para Mariano y el cacique Maicá, sus otros representados, brindando información detallada y precisa sobre los nombres de los linderos y la situación de los terrenos (con población, taperas, etc.) adyacentes como los anteriores y "por el Nor-Este-con otras suertes de tercera escala que también están pobladas cuyos nombres no recuerdo ahora”.

TABLA 1

Comparación de los puntos centrales de las tres solicitudes

\begin{tabular}{|l|l|l|l|}
\hline Expediente & 658 & 659 & 660 \\
\hline Fecha inicio & $04-02-1861$ & $04-02-1861$ & $04-02-1861$ \\
\hline Solicitante & Mariano Maicá & Juan Maicá & Antonio Medina \\
\hline Apoderado & Juan Cornell & Juan Cornell & Juan Cornell \\
\hline Suerte pedida & Fondos de la Suerte 30 & Fondos de la Suerte 31 & Fondos de la suerte 29 \\
\hline Testigo del interesado & José Flores & José Flores & José Flores \\
\hline Testigo de actuación & Basilio Vásquez & Basilio Vásquez & Basilio Vásquez \\
\hline Fecha resolución & 05-04-1861 & 05-04-1861 & 05-04-1861 \\
\hline Resultado & Negativo & Negativo & Negativo \\
\hline
\end{tabular}


De la información expuesta, colegimos que cinco años después de la firma del tratado y de la fundación de Villa Fidelidad en 1856, y dos años luego de la realización del plano y croquis en 1859, Cornell, importante autoridad militar de la frontera y residente en ese entonces en Azul, solicitó a pedido, y con poder especial otorgado por un instrumento público de sus tres poderdantes, tres suertes de estancia (una por cada uno) con arreglo al decreto de 1829 como representante legal ante la autoridad gubernamental en la ciudad de Buenos Aires. Cabe notar que sus tres poderdantes llevan en las fuentes la distinción de "Don", tal como el propio Cornell. Además, Mariano Maicá reviste exactamente el mismo altísimo grado militar que él como sargento mayor, nombrado en 1859. Además, el lenguaraz Antonio Medina, quien fue catalogado como "vecino" del pueblo de Azul, posiblemente guardaba relaciones de parentesco con Juan Medina, el "Capitán Mayor de los Indios Amigos” y con el “indio amigo" José María Medina, ambos donatarios originales de solares en Villa Fidelidad en 1856 (Lanteri, 2017).

Por su parte, sabemos que José Flores, quien firmó como testigo a ruego de los interesados porque ellos no sabían firmar, era un antiguo poblador de Azul, que se había casado con Tomasa Lemos en septiembre de 1848 (Uriarte de Louge, 2007, p. 67). En cuanto al testigo de actuación de los poderes, Basilio Vásquez, se trataba de un individuo natural de Buenos Aires, que fue registrado por Cornell como uno de los primeros pobladores donatarios de las suertes de estancia que habían sido otorgadas por Rosas en la frontera de Azul. Allí, aguas arriba del Fortín Estomba, Vásquez había formado en 1832 un establecimiento de estancia con plantíos que lindaba con el terreno en propiedad de Silva; dicho establecimiento fue destruido y su ganado hurtado en 1855 por un ataque indígena, por lo que pasó a convertirse en una tapera (Cornell, 1859a). En septiembre de 1842, este donatario se casó con Encarnación Navas en la parroquia de Azul (Uriarte de Louge, 2007, p.40). Su suerte de estancia fue escriturada en 1866 por Ignacio Rivas, otra importante autoridad militar de la frontera, y formaba parte de aquellas "destinadas para los Indios" (Azul, 1832-1875).

Estas cuestiones ratifican que todos ellos residían en el pueblo y el partido desde hacía tiempo, y que tenían vínculos cercanos y cotidianos de distinto tipo y envergadura, como el hecho de compartir acciones militares, espacios de sociabilidad, actividades productivas y económicas, etc., dentro de un alto grado de hibridación y mestizaje biológico, socioétnico y cultural propio de las zonas de frontera vinculadas, a su vez, con amplios circuitos de "tierra adentro" (Mayo, 2000; Mandrini, 2006; Farberman y Ratto, 2009).

Otro aspecto relevante a destacar es la importancia del servicio militar para la solicitud de las tierras. Cornell (1861) mencionó en varias oportunidades en su requerimiento tanto el grado militar señalado en Mariano Maicá como que él y la tribu liderada por su padre, el cacique Juan Maicá, se hallaban en actual servicio guareciendo la frontera de Azul. Este argumento utilizado por Cornell para validar los pedidos de terrenos va de la mano de la contraprestación de bienes por servicios públicos (tierras por servicio armado, en este caso) de raigambre anterior en la zona y en la campaña de Buenos Aires. Idénticas condiciones se habían producido en el caso de Villa Fidelidad cinco años antes con el mismo grupo étnico y durante el gobierno de Rosas con los vecinos milicianos "criollos" que poblaban esa zona de frontera (Lanteri, 201 1,2017) y también otras, como el oeste.

Además, con respecto a la modalidad de las suertes de estancia donadas en Azul, el decreto de 1829 que fue validado por la ley del 21/10/1857 durante el proceso de revisión liberal post-rosista, constituyó no sólo el pilar legal que permitió ordenar y clarificar los títulos de los terrenos, sino que también se erigió nada más y nada menos que en la normativa de base utilizada para procesar los mentados "premios por combates contra los indios" para toda la frontera provincial, otrora otorgados durante el gobierno de Rosas (Lanteri, 2017; Valencia, 2005).

El manejo de "las leyes de la materia" sobre tierras públicas en general y la modalidad de las donaciones condicionadas de suertes de estancia en Azul dentro del marco provincial, en particular, es asimismo destacable, tanto de parte de Cornell como de los propios indígenas. En este sentido, la cita del decreto de 1829 y la noción de las condiciones requeridas para la obtención de la propiedad titulada -el poblamiento 
efectivo, la puesta en producción del terreno y la obligación de cumplir el servicio armado en el punto (Lanteri, 2011) - fueron referidas y utilizadas como argumentos en la confección de los poderes así como en las solicitudes formales. Es significativo también notar aquí la voluntad referida por el cacique, su hijo y el lenguaraz, de poblar efectivamente o de constituir un establecimiento de estancia, lo que denota el conocimiento de antemano de las condiciones requeridas por la normativa, conjuntamente con la intención del poblamiento efectivo in situ y de forma colindante entre los tres interesados. Esto último permite suponer un uso colectivo de los terrenos por parte de sus familias y/o el resto de su parcialidad, más allá de que los pedidos hayan sido procesados a título individual, según los parámetros de la normativa vigente, como también fue destacado para los sectores oeste y extremo sur de la frontera coetánea (Literas, 2015; Martinelli, 2017).

Asimismo es dable señalar el notable saber "a ras del suelo" que Cornell tenía acerca de los pobladores originales, los posteriores, y sobre los terrenos. Para elaborar su informe en 1859, él mismo se apersonó directamente en la zona para averiguar, según la información de los vecinos y autoridades, la historia previa de cada suerte: los primeros pobladores, las enajenaciones o traspasos habidos, los eventuales robos y perjuicios de parte de indígenas o enemigos políticos, las actividades productivas, etc. De este modo, el apoderado sabía a ciencia cierta, de memoria o según sus propios registros, si las suertes pedidas estaban pobladas, eran taperas, tenían linderos, etc., o si eran campos baldíos susceptibles de ser denunciados por particulares al Estado.

¿Dónde estaban situados los terrenos solicitados y cuáles eran sus antecedentes? Fueron requeridos en segunda y tercera escala, es decir, a los fondos de otras suertes principales, núm. 29, 30 y 31, o sea, colindantes entre sí, de forma relativamente cercana a la ubicación del enclave urbano de Villa Fidelidad y bastante lejos del establecimiento de las "tolderías de Catriel" sobre el arroyo Nievas (Figura 2). Se encontraban inmejorablemente situadas sobre el Camino de Postas, marcado por Cornell en su croquis con la línea punteada, es decir, en una de las principales rutas comerciales - que era paso obligado de carretas, postas, mercachifles, pulperos y todo el flujo del comercio interétnico en la frontera-, que unía varios puntos de intercambio centrales y cercanos a las principales rastrilladas hacia Chile. Según el propio informe y plano de Cornell, la suerte ${ }^{\circ} 29$ había sido poblada con hacienda por Domingo Alvarado en 1832, es decir, en el mismo año de inicio de las donaciones en Azul, y se había vendido en 1838 a Pablo Alvarado, quien continuaba poblándola en 1859. La n 30 había sido establecida con poblaciones y ganado en 1834 por Evaristo Castro, quien en 1838 vendió también a la testamentaria Alvarado, que continuaba ocupándola en la fecha del informe. Finalmente, en la suerte $n^{\circ} 31$ Manuel Muñoz había formado un establecimiento en 1840, y aunque había sido robado por los indios algunas veces, continuaba poblándolo en 1859 (Cornell, 1859a, 1859b). 
FIGURA 2

Fragmento del "Plano del Partido Arroyo Azul construido por el Sargento Mayor Don Juan Cornell” (1859), donde figura la localización de las tres suertes solicitadas

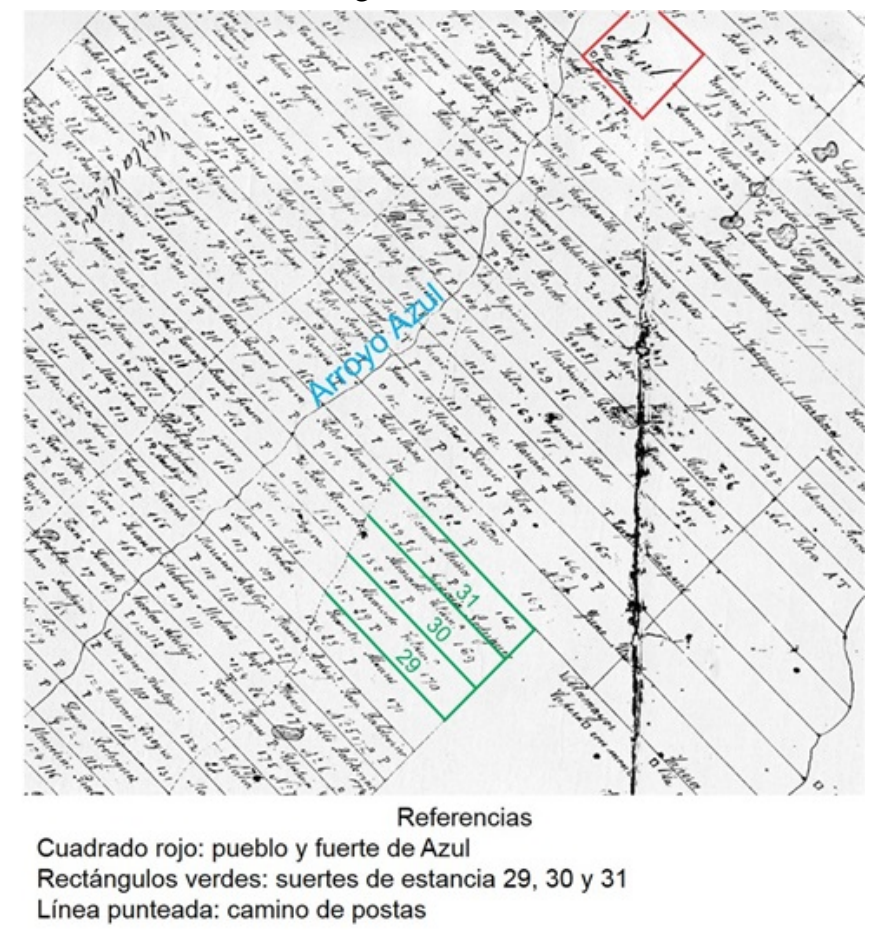

Empero, ¿qué fue de las solicitudes y cuál su desenlace? Todas corrieron la misma tramitación aludida en párrafos anteriores y vale detenerse en la información otorgada por el DT al jefe de la Oficina de Tierras Públicas para expedirse sobre las tres. Según decían los miembros que suscribieron, Salas, Moreno, Fernández y Malaver, en Buenos Aires el 19/03/1861, el terreno requerido

según los registros que obran en esta Oficina debe caer sino en el todo al menos en una parte dentro de los límites del terreno de propiedad de Dn Vicente Eladio y Dn Mariano Casares cuyo fondos se estiende desde el arroyo Tapalque donde tiene su frente, hacia los fondos de las suertes de Estancia del arroyo Azul.

\section{Y agrega entonces que:}

Esta circunstancia hace que esta solicitud no pueda tener lugar libremente en el parage q se indica-A mas de esto el Depto Topgo no sabe qe los campos á que se hace referencia esten destinados á darse en propiedad á los que hoy quieran ir á poblarlos, por que si bien en los campos del Arroyo Azul, hubo una porción destinados al objeto indicado, después de las leyes que se han dado sobre tierras publicas aquel objeto ha debido terminar.

El jefe de la Oficina de Tierras Públicas, Marcó del Pont, elevó el pedido cuatro días después para resolución al Gobierno, que se expidió rápidamente. De esta forma, el 5 de abril B. Mitre y J. Gelly y Obes, gobernador provincial y ministro interino de Guerra y Marina, respectivamente, informaron que:

Visto este espediente, lo informado por el Departamento Topográfico, y resultando que los terrenos que se solicitan recaen en todo ó en parte dentro de los limites del que posee en propiedad Don Vicente E y Don Marino Casares, y que á mas de esto el decreto de 19 de Setiembre de 1829 concedia suertes de estancias en el Azul á los que se poblasen en aquella época, no á los que después de treinta años se propusieron recién llenar aquel requisito, pues ya seria sin objeto dicha concesion, que tuvo en vista llevar poblacion a aquellos parajes, entonces completamente desiertos, y que ella solo puede hacerse efectiva con los que cumplieron inmediatamente las prescripciones de la referida disposicion, notifíquese a los interesados: Que no ha lugar a su solicitud. 
Recapitulando, el DT informó que los terrenos requeridos recaían en todo o en parte sobre la propiedad de otros dos particulares, por lo que la solicitud no podía tener lugar libremente allí. Además, que no sabía con certeza si los campos denunciados podían darse en propiedad como el resto de la suertes destinadas a los que en ese momento las pidiesen en función de las flamantes reglamentaciones respectivas. Ambos argumentos fueron utilizados y ampliados por el gobierno para denegar finalmente las solicitudes a los Maicá (Cornell, 1861). Con todo, ¿esto era realmente así? Veamos. En primer lugar, sobre Vicente Eladio y Mariano Casares, sabemos que los Casares sí habían denunciado y/o usufructuaban campos de forma coetánea en la región: Vicente E. en Azul y Tapalqué, Francisco y Sebastián también en este lugar y Lorenzo en Olavarría. Además, Mariano solicitó en arrendamiento al gobierno un campo de 16.199 hectáreas el 20/07/64 en Tres Arroyos; lo mismo hizo Vicente E. siete días después (Valencia 2005, p. 262). Ambos renovaron sus contratos en 1870 (Valencia, 2005, p. 300). Mediante la ley de 1871, Mariano compró esos terrenos en 1872 más 8.099 ha en Necochea al año siguiente; en tanto Vicente E. hizo lo mismo que su hermano en Tres Arroyos (Valencia, 2005, p. 348). Mariano figura como uno de los principales propietarios de la campaña, con una acumulación de 24.297 ha (Valencia, 2005, p. 358).

En segundo lugar, sobre la cuestión de las suertes de estancia, recordemos que la ley del 21/10/57, promulgada bajo la coyuntura de "ordenamiento legal" luego de la caída del gobierno de Rosas, autorizó el otorgamiento en propiedad de las suertes que habían sido donadas mediante el decreto de 1829 en caso de haber cumplido con los requerimientos de poblamiento, puesta en producción y servicio armado en la zona. Para ello los interesados debían acreditar un mínimo de 10 años de poblamiento efectivo e ininterrumpido, lo que produjo inmediatas y múltiples solicitudes de particulares al Estado y traspasos privados que continuaron hasta fines de siglo o incluso principios del XX (Lanteri, 2013). Por otro lado, esa ley fue solo el comienzo de las normativas que buscaban reglamentar el ordenamiento definitivo de esta peculiar modalidad de enajenación de la tierra pública a particulares en la campaña de Buenos Aires, que continuó luego con más decretos y reglamentaciones - muchas veces superpuestas, ambiguas o contradictorias entre sí- en la década de 1860, y posteriormente, sobre el plazo de cumplimiento de las condiciones que posibilitaban acceder a la propiedad titulada, la etnicidad de los pobladores habilitados para ello, ${ }^{10}$ entre otros aspectos que fueron incluso criticados por algunos coetáneos (Zinny, 1864).

Lo anterior implica que, si bien el DT y el gobierno eran los gestores y conocedores de esas disposiciones e información, esta cuestión aún se estaba intentando ordenar cuando fueron hechas las solicitudes de tierras analizadas -1861 - con la intervención de distintos agentes, autoridades, instituciones y jurisdicciones, finalizándose más tarde y con variaciones en su implementación legal en la práctica. Además, si bien hubo solicitudes desde 1857, la mayoría de las suertes comenzaron a ser escrituradas recién a partir de 1865, a favor de distintos pobladores, y el fisco siempre tendió a priorizar a los pobladores originales que pudieron acreditar efectivamente el cumplimiento de los requerimientos frente a los "antiguos enfiteutas", "arrendatarios actuales" o pobladores posteriores (Lanteri, 2013), lo que supone que se utilizaron los mismos criterios oficiales para dirimir el asunto que con el resto de la población no indígena. Antes de esa fecha, solo habían sido vendidas dos suertes y una parte de otra a Lino y Luis Pardo en 1861. En 1865, Pablo Muñoz escrituró la suerte $n^{\circ} 105\left(n^{\circ} 113\right.$ en el plano de Cornell, Figura 2) de acuerdo a la ley del 21/10/57, cercana a las solicitadas por los Maicá, la cual había sido poblada sobre la costa del arroyo en la temprana fecha de 1835 (Azul, 1832-1875; Cornell, 1859a).

\section{Conclusiones}

Las tres solicitudes de suertes de estancias analizadas en este trabajo resultan novedosas en varios sentidos. En primer lugar, porque se trata de expedientes producidos a partir de pedidos concretos, que reflejan de modo cabal la intención de los solicitantes de obtener tierras y que constituyen iniciativas que permiten enfocar la agencia indígena en cuanto al acceso a la tierra, aspecto que venimos destacando para los "indios amigos" en la frontera sur de Buenos Aires (Lanteri y Pedrotta, 2018). Si en el caso de Villa Fidelidad puede 
plantearse que el coronel M. Escalada tuvo un papel central en las gestiones y que, en algún modo, la dinámica que se planteó fue "de arriba hacia abajo", no quedan dudas que en el caso del cacique Juan Maicá, su hijo Mariano y el lenguaraz Medina, la dirección fue inversa: desde los agentes socioétnicos hacia el Estado, es decir, "de abajo hacia arriba", con la intermediación de Cornell.

En segundo lugar, la lectura minuciosa de los expedientes presentados pone claramente de manifiesto que se trata de la primera -y por lo que sabemos hasta ahora única- solicitud de suertes de estancia mediante la política de "donaciones condicionadas" por parte de indígenas, así como el aceitado conocimiento y manejo de la normativa y los procedimientos propios de las instituciones encargadas de administrar y resolver las cuestiones atinentes a las tierras por parte de un grupo de "indios amigos" del centro-sud bonaerense. En tal sentido, no se advierten diferencias con respecto a los pobladores criollos, quienes utilizaban el mismo utillaje institucional y normativo en sus requerimientos ante la autoridad. Asimismo, el tratamiento y la respuesta dados a sus solicitudes por parte de esta no parecen haber estado mediados por su condición étnica, sino que se atuvieron a las disposiciones legales vigentes para el conjunto social general. Estos elementos ratifican la capacidad de agencia de las sociedades indígenas, sin desconocer, ciertamente, los límites sobre la misma, que fueron imponiendo las condiciones de su relación con la sociedad estatal conforme se afianzaba el Estado nacional. La apropiación y el uso estratégico de los mecanismos legales vigentes, para obtener el acceso a la tierra que observamos en este caso, han sido también planteados con respecto a otros caciques "amigos": los Rondeau situados en el oeste bonaerense y los Ancalao, en la zona de Bahía Blanca, respectivamente (Literas, 2015; Martinelli y Acosta, 2016; Martinelli, 2017).

La mencionada utilización de los marcos jurídicos e institucionales vigentes para el acceso a la tierra se relaciona, asimismo, con la eventual obtención de derechos y el ejercicio de prácticas políticas asociadas con la ciudadanía armada y/o electoral. Algunos autores, como Quijada (2011), han planteado que durante las etapas de formación republicana, ciertas prácticas vinculadas con servicios a la patria, el bien común y la vecindad -como por ejemplo, el servicio armado- pudieron constituir una vía de acceso a la ciudadanía de los indígenas, junto a otros sectores sociales. Sin duda, este tema requerirá mayor profundización a futuro, ya que constituye una interesante vía de indagación sobre el desarrollo y ejercicio de diferentes tipos de derechos desde un punto de vista jurídico, político y sociocultural.

La intervención del sargento mayor Juan Cornell merece una mención aparte, ya que abre una cantidad de cuestiones a considerar. Por un lado, su trayectoria en la frontera sur evidencia relaciones de larga data con los grupos indígenas, tanto "amigos" como eventuales aliados y hostiles. Por otro, tuvo un desempeño destacado en actividades militares, topográficas y cartográficas, específicamente en el relevamiento de las suertes de estancia del Azul, hecho por pedido expreso de Gelly y Obes en 1859. Todo indica que era una autoridad con un conocimiento detallado de la evolución de cada uno de esos terrenos y de su situación al momento de producirse los pedidos de los Maicá, a punto tal que la información contenida en su plano -de difícil acceso- formó parte de la argumentación de los pedidos de tierra. Sin duda, Cornell era un "intermediario cultural" (Ares Queija y Gruzinski, 1997; Ratto, 2005), experto no solo en cuestiones generales de tierras, sino también con experiencia en la diplomacia interétnica y con un acabado conocimiento de la realidad azuleña -donde entonces residía - y del funcionamiento de las instituciones pertinentes. Hasta el momento, no hemos hallado otras solicitudes de tierras - por parte de indígenas o no indígenas- en las que Cornell haya intervenido como apoderado, lo que exige prestar atención en adelante a las circunstancias que motivaron su actuación.

En tercer lugar, el caso analizado muestra la estrecha relación que existió entre la prestación de servicios -como fuerzas militares- por parte de los "indios amigos" y sus posibilidades de acceso a la tierra, lo que robustecía un vínculo que se ha observado también en relación con distintas agrupaciones, en otros sectores de la frontera bonaerense y bajo coyunturas y normativas diferentes (de Jong, 2015; Literas, 2015; Literas y Barbuto, 2015; Martinelli y Acosta, 2016; entre otros). El elemento clave para comprender el otorgamiento 
y/o el reconocimiento de tierras a los indígenas por parte del Estado radica, sin duda, en las coyunturas bélicas que atravesaron el período que se inició con la caída de Rosas hasta la definitiva consolidación del EstadoNación. En el contexto crítico del giro en la política indígena del Estado de Buenos Aires y de la violencia interétnica en la frontera sur, las cuales se desplegaron justamente a mediados de la década de 1850 , hemos sostenido que se llevaron a cabo estrategias diferentes de negociación, tanto por parte de las autoridades militares y políticas como por los distintos caciques que integraron la heterogénea tribu de "indios amigos" liderada por J. Catriel y J. M. Cachul. Buen ejemplo de estas diferencias fue la concreción simultánea del tratado de paz de 1856, en el cual se reconoció por escrito, y por vez primera, la "legítima propiedad" de tierras a indígenas en Buenos Aires durante el período independiente y la fundación de Villa Fidelidad en un sector aledaño al pueblo de Azul, cuyos solares fueron inicial y principalmente adjudicados a los integrantes de la parcialidad al mando del cacique Maicá (Lanteri y Pedrotta, 2018).

Así, las fuerzas indígenas -ya sea regulares o como apoyos ocasionales- y las tierras en sus diversas modalidades - comunales, individuales, rurales, urbanas, etc.- fueron elementos centrales en la pacificación de la frontera bonaerense entre 1856 y 1857, y también, según sugieren las fuentes analizadas en este trabajo, en los convulsionados meses que se sucedieron entre las batallas de Cepeda (1859) y Pavón (1861). Recordemos que las solicitudes de tierras fueron tramitadas entre febrero y abril y la batalla de Pavón ocurrió en septiembre de ese año. ${ }^{11}$ Los solicitantes fueron tres personajes notables dentro del grupo de los Maicá, que se caracterizó por su sostenida lealtad y prestación de servicios militares al Estado de Buenos Aires en comparación a otros grupos de "indios amigos" del centro-sur bonaerense. Sin duda, el período de unificación nacional, que comenzó luego de Pavón y con la presidencia de B. Mitre en 1862, marcó otro rumbo en la política indígena y condiciones diferentes en la frontera bonaerense, en cuyas nuevas coordenadas será necesario situar los posteriores pedidos y otorgamientos de tierras a la población indígena en la frontera sur de Buenos Aires, así como comparar la evidencia con otros sectores.

Los datos originales obtenidos a partir del análisis de las solicitudes de las suertes de estancia efectuadas por el cacique Maicá, su hijo Mariano Maicá y su lenguaraz, junto a la nueva documentación recabada, permiten repensar la adscripción de la parcialidad de Maicá dentro de la "gran tribu catrielera", si consideramos a partir de qué momento y por qué comenzaron a constituirse en agentes con un alto grado de independencia en la negociación de las condiciones bajo las cuales se desempeñaron como "indios amigos". En este aspecto, además de las necesidades militares oficiales, debemos tener en cuenta el rasgo competitivo y segmental de la organización social y política indígena (Bechis, 1989; de Jong, 2011). Es indudable que la trayectoria del linaje Maicá es contrastante con los demás caciques de diferente jerarquía que integraron en distintos momentos la agrupación catrielera al mando de los caciques principales Catriel y Cachul, así como el trato particular que recibió por parte de los vecinos, las autoridades militares y la municipalidad de Azul. Este mismo trato deferente hacia los Maicá ha sido subrayado en lo concerniente al ámbito de la justicia (Gambetti, 2014). Como hemos sostenido en trabajos anteriores, los Maicá gestionaban y recibían su paga, armas y racionamiento de forma separada de los demás “indios amigos” locales (Lanteri y Pedrotta, 2018).

También es relevante marcar que, si bien el gobierno provincial denegó las tres suertes de estancia que fueron solicitadas en 1861, como en muchos otros casos a otros pobladores criollos por las razones mencionadas, ${ }^{12}$ dos años después la Corporación Municipal de Azul "acordó darles á los Indios del Casique Maicá un sitio solar del otro lado del Arroyo pagando cada uno de estos veinte pesos $\mathrm{m} / \mathrm{c}$ al Agrimensor" (Azul, 1863). Esto podría evidenciar que los indígenas tenían mayores posibilidades relativas de gestionar y obtener la tierra a nivel municipal que provincial, como sucedió con el caso de Villa Fidelidad, al encontrarse en la traza urbana (Lanteri, 2017). De hecho, las municipalidades de campaña creadas desde mediados de la década de 1850 generaron tensiones entre las esferas de poder, autoridades y jurisdicciones político-institucionales de los ámbitos municipal, provincial y nacional, lo que afectó tanto las tierras como otras cuestiones, que fueron objeto de complejos reacomodamientos desde entonces. 
Por último, otro tema novedoso que se abre con el caso de los Maicá en Azul refiere a una organización territorial a partir de un sistema que podríamos llamar de doble asentamiento, que combinaba la residencia en el pueblo con el trabajo dedicado a la producción agropecuaria en el ámbito rural, y que se ha planteado para otros "indios amigos" de la frontera oeste bonaerense (Literas 2015, p. 71). Nos preguntamos si los tres pedidos de tierras analizados en este trabajo no están expresando justamente la intención de concretar ese tipo de asentamiento por parte de la parcialidad de Maicá, línea de indagación que esperamos continuar a futuro.

\section{Agradecimientos}

Este trabajo fue realizado en el marco de los proyectos PIP "Arqueología e historia de la frontera sur de Buenos Aires durante la segunda mitad del siglo XIX: campos, ejidos y territorio indígena desde una mirada interdisciplinar", financiado por el CONICET y PICT "La construcción del territorio en la frontera sur de Buenos Aires en perspectiva arqueológica e histórica. Campos, ejidos y tierras indígenas en la segunda mitad del S. XIX", financiado por la ANPCyT. V. P. agradece a la Fundación Félix de Azara y la Universidad Maimónides por el apoyo institucional. Finalmente, ambas autoras agradecemos a Vanesa Bagaloni por su colaboración con las imágenes y a los evaluadores, cuyas observaciones permitieron mejorar el manuscrito.

\section{Referencias BibliográficAs}

AA.VV. (2015). Dossier "Tierras comunales e indivisas en las pampas y el Noroeste Argentino (siglos XVII-XIX)". Revista de Ciencias Sociales, 7(27), 7-117. Recuperado de https://ridaa.unq.edu.ar/handle/20.500.11807/1611

Arena, J., Cortés, J., y Valverde, A. (1967). Ensayo histórico del Partido de Olavarría. Olavarría: Municipalidad de Olavarría.

Ares Queija, B., y Gruzinski, S. (coords.). (1997). Entre dos mundos. Fronteras culturales y agentes mediadores. Sevilla: EEHA, CSIC.

Azul. (1832-1875). Libros de Suertes del Arroyo Azul 1832-1875. Número 253, Folio 105, Archivo Histórico, Dirección de Geodesia y Catastro, Ministerio de Infraestructura y Servicios Públicos de la Provincia de Buenos Aires, Argentina.

Azul. (1863). Actas de Sesiones Municipales. Sesión del 22 de junio de 1863, página 183, Museo Etnográfico y Archivo Histórico Enrique Squirru, Azul, Provincia de Buenos Aires, Argentina.

Barbará, F. (1856). Usos y costumbres de los indios pampas.

Barcos, M. F. (2017). Tratos, batallas y malones. El accionar indígena en la Frontera Sur durante el Sitio a la ciudad de Buenos Aires. Corpus, 7(1), 1-30. Recuperado de https://journals.openedition.org/corpusarchivos/1871

Bechis, M. (1989). Los lideratos politicos en el área arauco-pampeana en el siglo XIX: ¿autoridad o poder? Ponencia presentada en el I Congreso de Etnohistoria, julio 17-21. Buenos Aires, Argentina.

Canciani, L. (2017). Frontera, militarización y politica armada. La Guardia Nacional de la provincia de Buenos Aires durante la construcción del Estado Nacional (1852-1880). La Plata: AAAHPBA.

Catriel, J. (1859). Carta al Gobernador Valentín Alsina. Comandancia de Fronteras, Legajo 20-2-2, Sala X, Archivo General de la Nación Argentina.

Comandancia (1859). Comandancia General de la Frontera Sud. Azul, 12 de abril de 1859, Comandancia de Fronteras, Legajo 20-2-2, Sala X, Archivo General de la Nación Argentina.

Cordero, G. (2017). Territorialidad y política en Salinas Grandes (décadas de 1860 y 1870). Pasado Abierto, 5, 91-114. Recuperado de https://fh.mdp.edu.ar/revistas/index.php/pasadoabierto/article/view/2191

Cornell, J. (1859a). Informe dirigido al Sor Ministro de Gobierno en el que se da cuenta haber desempeñado Don Juan Cornell la comisión de que fue encargado para el Partido Arroyo Azul. Archivo Histórico, Dirección de Geodesia y Catastro, Ministerio de Infraestructura y Servicios Públicos de la Provincia de Buenos Aires, Argentina. 
Cornell, J. (1859b). Plano del Partido Arroyo Azul construidoporel Sargento Mayor Don Juan Cornell. Plano 1270-29-3, Archivo Histórico, Dirección de Geodesia y Catastro, Ministerio de Infraestructura y Servicios Públicos de la Provincia de Buenos Aires, Argentina.

Cornell, J. (1861). Cornell, don Juan por don Mariano Mayca solicita una suerte de estancia en el Azul. Legajo 107, Número 8814, Escribanía Mayor de Gobierno, Archivo Histórico de la Provincia de Buenos Aires, Argentina.

Cornell, J. [1864] (1995). "...De los hechos de armas con los indios". Informe solicitado por el ministro de Guerra y Marina General don Juan Andrés Gelly y Obes. En B. Goldwasser y C. Cansanello, (eds.). Fuentes para el estudio de la historia de la provincia de Buenos Aires(pp. 31-48). Luján-Tandil: UNLu/IEHS-UNICEN.

de Jong, I. (2011). Funcionarios de dos mundos en un espacio liminal. Tefros, 9(1-2), 1-37. Recuperado de http://ww w2.hum.unrc.edu.ar/ojs/index.php/tefros/article/view/238

de Jong, I. (2015). El acceso a la tierra entre los indios amigos de la frontera bonaerense (1850-1880). Revista de Ciencias Sociales, 7(27), 87-117. Recuperado de https://ridaa.unq.edu.ar/handle/20.500.11807/1611

de Jong, I., y Escobar Ohmstede, A. (coords.). (2016). Las poblaciones indigenas en la conformación de las naciones y los Estados en la América Latina decimonónica. México: Colmex-Colmich-CIESAS.

Del Valle, A. (1926). Recordando al pasado. Campañas por la civilización. Tomo I.

Escobar Ohmstede, A., Medina Bustos, J., y Trejo Contreras, Z. (coords.) (2015). Los efectos del liberalismo en México. Siglo XIX. México: El Colegio de Sonora- CIESAS.

Expediente (1858). Expediente iniciado por la Comisión de Hacendados. Comandancia de Fronteras, Legajo 19-8-4, Sala X, Archivo General de la Nación Argentina.

Farberman, J., y Ratto, S. (2009). Historias mestizas en el Tucumán colonial y las pampas (siglos XVII-XIX). Buenos Aires: Biblos.

Gambetti, N. (2014). Los indios amigos de Buenos Aires y la administración de justicia (1852-1870). Ponencia presentada en la IV Jornada de becarios y tesistas 2014, Departamento de Ciencias Sociales, UNQ. Recuperado de http://sociales.unq.edu.ar/wp-content/uploads/byt2014/ponencias/eje08/GambettiNadia-Losindiosamig osdeBuenosAiresylaAdministraci\%C3\%B3ndeJusticia1852-1870.pdf

Gelly y Obes, J. 1860. Carta del Ministro de Guerra y Marina al Inspector General de Armas. Frontera con los Indios, Documento 1434, Servicio Histórico del Ejército, Argentina.

Gelly y Obes, J. 1861. Carta del Ministro de Guerra y Marina al Inspector General de Armas. Frontera con los Indios, Documento 8638, Servicio Histórico del Ejército, Argentina.

Goldwasser, B., y Cansanello, C. (1995). Fuentes para el estudio de la historia de la provincia de Buenos Aires. LujánTandil: UNLu/IEHS-UNICEN.

González, M. A. (1967). Catrie Mapu-Monografía sobre los Catriel. Olavarría: Museo Etnográfico Municipal Dámaso Arce.

Hux, M. (1993). Caciques Puelches, Pampas y Serranos. Buenos Aires: Marymar. Lanteri, S. (2011). Un vecindario federal. La construcción del orden rosista en la frontera sur de Buenos Aires (Azul y Tapalqué). Córdoba: Centro de Estudios Históricos "Prof. Carlos Segreti".

Lanteri, S. (2011). Un vecindario federal. La construcción del orden rosista en la frontera sur de Buenos Aires (Azul y Tapalqué). Córdoba: Centro de Estudios Históricos "Prof. Carlos Segreti”.

Lanteri, S. (2013). Reformas liberales, sociedad rural y derechos de propiedad territorial en la frontera sur bonaerense ( $2^{a}$ mitad del siglo XIX). Las 'donaciones condicionadas' de Azul. Trabajos y Comunicaciones, 39, 1-27. Recuperado de http://www.trabajosycomunicaciones.fahce.unlp.edu.ar/article/view/TyC2013n39a04/pdf_4

Lanteri, S. (2017). De la inmensidad de la llanura al arrabal de un pueblo. Villa Fidelidad, los premios y donaciones de tierras en la frontera sur bonaerense durante el proceso de revisión liberal. Pasado Abierto, 3(6), 177-200. Recuperado de http://fh.mdp.edu.ar/revistas/index.php/pasadoabierto/article/view/2490

Lanteri, S., y Pedrotta, V. (2012). Mojones de piedra y sangre en la pampa bonaerense. Estado, sociedad y territorio en la frontera sur durante la segunda mitad del siglo XIX. Tefros, 10(1-2), 1-25. Recuperado de http://www2.hum .unrc.edu.ar/ojs/index.php/tefros/article/view/255 
Lanteri, S., y Pedrotta, V. (2018). Tierras, armas y política en la frontera sur bonaerense durante la década de 1850. Los `indios amigos', Maicá y Villa Fidelidad, Anuario del Instituto de Historia Argentina, 18(1), 1-21. https:// doi.org/10.24215/2314257Xe066

Lanteri, S., Ratto, S., de Jong, I., y Pedrotta, V. (2011). Territorialidad indígena y políticas oficiales de colonización. Los casos de Azul y Tapalqué en la frontera sur bonaerense (siglo XIX). AntíTeses, 4(8), 729-752. Recuperado de https://www.uel.br/revistas/uel/index.php/antiteses/article/download/.../9470

Levaggi, A. (2000). Paz en la Frontera. Buenos Aires: Universidad del Museo Social.

Literas, L. (2015). De donaciones, arrendamientos y compras. Acceso y uso de la tierra de los indios amigos (la tribu de Rondeau, segunda mitad siglo XIX). Publicar, XIII(XVIII), 59-84.

Literas, L., y Barbuto. L. (2015). El acceso a la tierra de los indios amigos. Una comparación preliminar de las tribus de Catriel y Rondeau (Buenos Aires, segunda mitad del siglo XIX). Tefros, 13(2), 1-22. Recuperado de http:// www2.hum.unrc.edu.ar/ojs/index.php/tefros/article/view/335

Mandrini, R. (ed.). (2006). Vivir entre dos mundos. Conflicto y convivencia en las fronteras del sur de la Argentina. Siglos XVIII y XIX. Buenos Aires: Taurus.

Martinelli, M. L., y Acosta, M. (2016). La cuestión de la tierra pública en la frontera sur bonaerense en las décadas de 1860 y 1870: tensiones, negociaciones y agencias de indios, criollos e inmigrantes. El caso de Bahía Blanca. Tefros, 14(2), 87-156. Recuperado de http://www2.hum.unrc.edu.ar/ojs/index.php/tefros/article/view/453

Martinelli, M. L. (2017). Construcción estatal e "indios amigos": el acceso a la tierra de la tribu de Ancalao en el enclave fronterizo de Bahía Blanca". Memoria Americana, 25(1), 97-114.

Mayo, C. (2000). Vivir en la frontera. La casa, la dieta, la pulpería, la escuela (1770-1870). Buenos Aires: Biblos.

Motta, M., y Piccolo, M. (orgs.) (2017a). O Dominio de outrem. Propriedades e direitos no Brasil (Séculos XIX e XX). Vol. 2, Sao Luis: EDUEMA.

Motta, M., y Piccolo, M. (orgs.). (2017b). O Dominio de outrem. Posse epropriedade na Era Moderna (Portugale Brasil). Vol. 1, Sao Luis: EDUEMA.

Pedrotta, V., y Lanteri, S. (2016). Tierras y nuevas territorialidades indígenas en la frontera sur de Buenos Aires. Argentina. Historia y Arqueología de Villa Fidelidad (siglos XIX-XXI). Ponencia presentada en el Segundo Congreso Internacional Los pueblos indígenas de América Latina, Siglos XIX-XXI, septiembre 20-24, en Santa Rosa, Argentina.

Quijada, M. (2011). La lenta configuración de una "ciudadanía cívica" de frontera. Los indios amigos de Buenos Aires, 1820-1879 (con un estudio comparativo Estados Unidos-Argentina). En M. Quijada (ed.), De los cacicazgos a la ciudadania. Sistemas politicos en la frontera. Rio de la Plata, siglos XVIII-XX (pp. 149-289). Berlín: VGM.

Ratto, S. (2003). Una experiencia fronteriza exitosa: el 'negocio pacífico' de indios en la provincia de Buenos Aires (1829-1852). Revista de Indias, LXIII, 191-222.

Ratto, S. (2005). Caciques, autoridades fronterizas y lenguaraces: intermediarios culturales e interlocutores válidos en Buenos Aires (primera mitad del siglo XIX). Mundo Agrario, 10(5), 1-28. Recuperado de https://www.mundo agrario.unlp.edu.ar/article/view/v05n10a08

Ratto, S. (2015). Redes politicas en la frontera bonaerense (1836-1873). Crónica de un final anunciado. Bernal: UNQ. Recuperado de http://unidaddepublicaciones.web.unq.edu.ar/wpcontent/uploads/sites/46/2016/04/Redes-p oliticas-e-book.pdf

Ronco, B. (1930). Cuentas de proveedores. Azul. Revista de Ciencias y Letras, 1(3), 141-144.

Sarramone, A. (1993). Catriel y los indios pampas de Buenos Aires. Azul: Biblos.

Uriarte de Louge, S. (2007). Transcripción de las partidas de matrimonios de la parroquia de Nuestra Señora del Rosario de Azul (provincia de Buenos Aires). La Plata: Instituto de Estudios Genealógicos y Heráldicos de la Provincia de Buenos Aires.

Valencia, M. (2005). Tierras públicas. Tierras privadas. Buenos Aires, 1852-1876. La Plata: AHPBA-UNLP.

Walther, J. C. (1973). La Conquista del Desierto. Buenos Aires: Eudeba. 
Yuln, M. (2017). Pueblos indígenas en la frontera bonaerense. Los asentamientos de "indios amigos" durante el siglo XIX. Scripta Nova, XXI(554), 1-32. Recuperado de http://revistes.ub.edu/index.php/ScriptaNova/article/vie $\mathrm{w} / 17384$

Zinny, A. (1864). Reseña relativa a las suertes de estancia ofrecidas en propiedad en el partido del Azul con citación de leyes $y$ decretos. Buenos Aires: Imprenta de Pablo E. Coni.

\section{Notas}

1 Utilizamos este concepto respetando las formas de autoadscripción provenientes de los documentos escritos de la época, sin connotaciones esencialistas o evolucionistas sobre la organización social indígena. Remitimos, entre otros, al clásico estudio de Bechis (1989) sobre el tema.

2 La obra de Walther hace una recopilación minuciosa -desde una perspectiva netamente militar- de los ataques y enfrentamientos de la coyuntura post-rosista, así como de los cambios en la estructura y organización de las fuerzas militares (Walther, 1973, pp. 285-311). Interesa destacar al respecto la participación indígena en el ejército. Así, los “indios amigos" liderados por el cacique Lucio López integraban la Primera División y los del cacique Francisco Ancalao formaban parte de la Segunda División, mientras que los “indios amigos" al mando de Juan Catriel y Juan Cachul se contabilizaban como una fuerza aparte.

3 Entre otras maniobras para alejar a algunos caciques segundos y capitanejos del liderazgo de los caciques principales, Rivas logró que el cacique Lucio López - al mando de unos 300 indígenas- se separara de la órbita de J. Catriel tiempo después (Hux, 1993; Sarramone, 1993). Luego del asesinato del primero en 1863, Chipitruz, su hijo y sucesor quedó al frente de un grupo independiente del liderazgo de Catriel y Cachul, que se estableció en las inmediaciones de la laguna Blanca Grande (Archivo Mitre, Tomo XXIV, p. 119, Carta de Chipitruz al Presidente Bartolomé Mitre, Azul, 1ro. de agosto de 1863).

4 El registro detallado de estas acciones puede consultarse en Hux (1993) y Sarramone (1993)

5 Sobre este tema puede consultarse Canciani (2017) y Barcos (2017).

6 Usamos aquí el término "catrielero" para designar, en sentido amplio, a la agrupación de "indios amigos", heterogénea, numerosa y de composición cambiante, que respondía al liderazgo de linaje de los caciques Catriel.

7 El "negocio pacífico de indios" constituyó una política cimentada durante el gobierno de Rosas de contraprestaciones de bienes y servicios públicos entre el gobierno y distintos grupos de "indios amigos", que se establecían en las cercanías de los pueblos o fuertes conforme se expandía la frontera, prestando esencialmente servicio armado (Ratto, 2003). Luego de la caída de Rosas, esta política fue reformulada (ver de Jong 2011 y Ratto 2015).

8 Vale detenerse en su biografía. Juan Cornell nació y murió en la ciudad de Buenos Aires, en 1798 y 1887 respectivamente y desempeñó diversas funciones político-militares. Fue alcalde de Kaquel-Huincul en 1823, miliciano y participante de campañas como la expedición a la sierra de la Ventana en 1824 y juez de paz de Monsalvo en 1826. Luego de desempeñar otros cargos, hacia 1852 fue agregado a la Plana Mayor Activa y en 1854 el general Bartolomé Mitre, merced a los trabajos topográficos que había efectuado durante sus campañas militares, lo incorporó al Instituto Histórico y Geográfico. Dentro de este realizó dos informes: por pedido del ministro de Gobierno en 1859, Dalmacio Vélez Sarsfield, sobre las "suertes de estancia" del arroyo Azul, y el de 1864, solicitado por el Ministro de Guerra y Marina Gelly y Obes, donde narró los acontecimientos más significativos de las campañas contra los pueblos indígenas (Goldwasser y Cansanello, 1995).

9 Cada suerte de estancia era equivalente a 2.025 has y representaba una unidad mediana en el contexto regional que, según los parámetros coetáneos, permitía la reproducción familiar (Lanteri, 2011).

10 Una de las cuestiones principales de debate fue si los extranjeros estaban habilitados para acceder a la propiedad como los criollos, aunque no se dijo nada de forma explícita sobre los indígenas en la normativa, no así en el plano de la praxis en la frontera, donde las autoridades locales tuvieron un rol central (Lanteri, 2013, 2017).

11 Vale recordar que el cumplimiento del servicio armado en la zona era uno de los requisitos fundamentales para la obtención de la propiedad según la ley de 1857 con base en los decretos de 1829 y 1832.

12 Encontramos similitudes sobre el criterio empleado para otorgar o no las tierras en el caso de los Ancalao en la zona de Bahía Blanca, en cuanto en 1863 el pedido de una suerte de estancia para Francisco Ancalao fue concedido porque no había solicitudes de terceros, mientras el pedido de Pedro Ancalao no fue concedido porque sí los había (Martinelli y Acosta, 2016; Martinelli, 2017). 